\title{
Regorafenib suppresses sinusoidal obstruction syndrome in rats.
}

\section{$\operatorname{AUTHOR}(\mathrm{S})$ :}

Okuno, Masayuki; Hatano, Etsuro; Nakamura, Kojiro; Miyagawa-Hayashino, Aya; Kasai, Yosuke; Nishio, Takahiro; Seo, Satoru; Taura, Kojiro; Uemoto, Shinji

\section{CITATION:}

Okuno, Masayuki ...[et al]. Regorafenib suppresses sinusoidal obstruction syndrome in rats.. The Journal of surgical research 2015, 193(2): 693-703

\section{ISSUE DATE:}

2015-02

URL:

http://hdl.handle.net/2433/193267

\section{RIGHT:}

C 2015 Elsevier Inc.; この論文は出版社版でありません。引用の際には 出版社版をご確認ご利用ください。; This is not the published version. Please cite only the published version. 
Regorafenib suppresses sinusoidal obstruction syndrome in rats

Masayuki Okuno, $\mathrm{MD}^{1}$, Etsuro Hatano, $\mathrm{MD}, \mathrm{PhD}^{1}$, Kojiro Nakamura, $\mathrm{MD}, \mathrm{PhD}^{1}$, Aya Miyagawa-Hayashino, MD, $\mathrm{PhD}^{2}$, Yosuke Kasai, $\mathrm{MD}^{1}$, Takahiro Nishio, $\mathrm{MD}^{1}$, Satoru Seo, $\mathrm{MD}^{1}$, PhD, Kojiro Taura, $\mathrm{MD}, \mathrm{PhD}^{1}$ and Shinji Uemoto, $\mathrm{MD}, \mathrm{PhD}^{1}$

1) Department of Surgery, Graduate School of Medicine, Kyoto University, Kyoto, Japan 54 Kawahara-cho, Shogoin, Sakyo-ku, Kyoto, Japan.

2) Department of Diagnostic Pathology, Kyoto University Hospital, Kyoto, Japan 54 Kawahara-cho, Shogoin, Sakyo-ku, Kyoto, Japan.

Financial support:

The authors received no financial support from funds or societies for this study.

Conflict of interest:

The authors report no proprietary or commercial interest in any product mentioned or concept discussed in this article.

Corresponding author:

Etsuro Hatano, MD, $\mathrm{PhD}$

Department of Surgery, Graduate School of Medicine Kyoto University

54 Kawahara-cho, Shogoin, Sakyo-ku, Kyoto, Japan. 
Tel: +81-75-751-4323, Fax: +81-75-751-4263, E-mail: etsu@kuhp.kyoto-u.ac.jp

Author contributions:

Conception and design of the study: M.O., E.H., K.N., S.S., K.T. and S.U.

Designed and performed experiments: M.O., K.N., A.M.-H., Y.K., and T.N.

Analyzed data: M.O., K.N. and A.M.-H.

Drafting of the article: M.O.

Critical revision: E.H., K.N. and S.U.

Final approval of the article: M.O., E.H. and S.U. 


\section{Abstract}

Background: Sinusoidal obstruction syndrome (SOS), a form of drug-induced liver injury related to oxaliplatin treatment, is associated with postoperative morbidity after hepatectomy. This study aimed to examine the impact of regorafenib, the first small-molecule kinase inhibitor to show efficacy against metastatic colorectal cancer, on a rat model of SOS.

Methods: Rats with monocrotaline (MCT)-induced SOS were divided into 2 groups according to treatment with either regorafenib $(6 \mathrm{mg} / \mathrm{kg})$ or vehicle alone, which were administered at 12 and $36 \mathrm{~h}$, respectively, before MCT administration. Histopathological examination and serum biochemistry tests were performed $48 \mathrm{~h}$ after MCT administration. Sinusoidal endothelial cells (SECs) were evaluated by immunohistochemistry and electron microscopy. To examine whether regorafenib preserved remnant liver function, a 30\% hepatectomy was performed in each group.

Results: The rats in the vehicle group displayed typical SOS features, whereas these features were suppressed in the regorafenib group. The total SOS scores were significantly lower in the regorafenib group than in the vehicle group. Immunohistochemistry and electron microscopy showed that regorafenib had a protective effect on SECs. The postoperative survival rate after 7 days was significantly better in the regorafenib group than in the vehicle group $(26.7 \%$ vs. $6.7 \%, \mathrm{p}<0.05)$. Regorafenib reduced the phosphorylation of extracellular signal-regulated kinase (ERK), which induced MMP-9 activation and decreased the activity of matrix metalloproteinase-9 (MMP-9), one of the crucial mediators of SOS development. 
Conclusions: Regorafenib suppressed MCT-induced SOS, concomitant with attenuating ERK phosphorylation and MMP-9 activation, suggesting that regorafenib may be a favorable agent for use in combination with oxaliplatin-based chemotherapy.

Keywords:

colorectal liver metastasis, kinase inhibitor, drug-induced liver injury, metalloproteinase-9, extracellular signal-regulated kinase 


\section{Introduction}

The liver is the most frequent site of metastasis from colorectal cancer $(1,2)$. Surgical resection is considered the only curative therapy for colorectal liver metastasis (CLM) $(3,4)$; however, only $25 \%$ of patients are eligible for hepatic resection with a curative intent $(5,6)$. Recently, new anticancer drugs including oxaliplatin have improved treatment results for colorectal cancer and enabled patients with initially unresectable CLM to undergo hepatic resections after neoadjuvant chemotherapy (7). Despite its benefits, however, preoperative oxaliplatin-based chemotherapy for CLM induces damage in the nontumorous liver parenchyma, known as sinusoidal obstruction syndrome (SOS) $(8,9)$, which is characterized by sinusoidal dilatation, endothelial damage in the central vein, and coagulative necrosis of hepatocytes (9). In patients with CLM, SOS contributes to postoperative morbidity after hepatectomy (10); therefore, a strategy to control SOS needs to be developed.

In a rat model of monocrotaline (MCT)-induced SOS, matrix metalloproteinase-9 (MMP-9) was described as a key mediator in the development of SOS $(11,12)$. In fact, MMP inhibitors have been shown to block the progression of SOS in the animal model, but the detailed mechanism has not been elucidated (12). We previously reported that sorafenib, a multikinase inhibitor, also attenuated MCT-induced SOS via MMP-9 suppression (13). However, a phase IIb study did not show a benefit in progression-free survival with the addition of sorafenib to oxaliplatin-based chemotherapy (14). Therefore, further clinical trials of sorafenib for colorectal cancer have not been conducted, indicating that future application of sorafenib for CLM is unlikely. 
Regorafenib is a novel oral multikinase inhibitor that targets various protein kinases such as vascular endothelial growth factor receptors (VEGFR) 1/2/3, tyrosine kinase with immunoglobulin and epidermal growth factor homology domain 2 (TIE-2), platelet-derived growth factor receptor-beta (PDGFR-beta), c-kit, ret, raf-1, and B-RAF (15). An international phase 3 trial (CORRECT) demonstrated a statistically significant improvement in overall survival and progression-free survival when regorafenib was administered to patients with metastatic colorectal cancer that progressed after all other approved standard therapies (16). On the basis of this trial, regorafenib was approved for metastatic colorectal cancer in several countries, including the US, Canada, Europe, and Japan. Furthermore, a phase $1 \mathrm{~b}$ study of combined therapy with regorafenib and oxaliplatin-based chemotherapy has been reported (17). Wilhelm et al. showed that regorafenib inhibited the phosphorylation of extracellular signal-regulated kinase (ERK), which is reported to be necessary for MMP-9 activation $(15,18)$. Thus, regorafenib also has the potential to attenuate SOS, similarly to sorafenib. However, it would be premature to conclude that regorafenib has a protective effect against SOS in the way sorafenib does, because the structure of regorafenib differs from that of sorafenib, resulting in a different biochemical profile from sorafenib (15).

The purpose of the present study was to examine the impact of regorafenib on SOS in a rat model and to clarify whether regorafenib improves mortality after hepatic resection.

\section{Materials and Methods}

\subsection{Reagents}


Monocrotaline (MCT) was purchased from Sigma-Aldrich (St. Louis, MO, USA). A solution of MCT was prepared at $10 \mathrm{mg} / \mathrm{mL}$, as previously reported (19). Regorafenib, a multikinase inhibitor, was prepared as a solution in polypropylene glycol/PEG400/Pluronic F68 (42.5/42.5/15 + 20\% Aqua). The regorafenib compound (kindly provided by Bayer HealthCare Pharmaceuticals Inc. [Whippany, NJ, USA]) was stored at room temperature in the dark (15). Polypropylene glycol and PEG400 were purchased from Wako Pure Chemical Industries (Osaka, Japan). Pluronic F68 was purchased from Sigma-Aldrich.

\subsection{Animals}

We used 7-9-week-old male Sprague-Dawley rats (SLC, Shizuoka, Japan) weighing 250-350 g. The animals had free access to food and water. All experiments were conducted in accordance with the guidelines for the care and use of laboratory animals of the animal research committee of Kyoto University. The animals received humane care in accordance with the NIH Guidelines for the Care and Use of Laboratory Animals.

\subsection{Experimental protocol}

MCT-treated rats were used as the experimental model of SOS $(13,20,21)$. Rats were fasted for $12 \mathrm{~h}$ before oral administration of MCT, but had free access to water. MCT (90 $\mathrm{mg} / \mathrm{kg}$ ) was administered by gavage, after which the rats were allowed to consume food and water ad libitum. To evaluate the efficacy of regorafenib on SOS, the rats were divided into 2 groups ( $\mathrm{n}=20$ in each group) according to treatment with regorafenib $(6 \mathrm{mg} / \mathrm{kg})$ or vehicle, by oral gavage, $12 \mathrm{~h}$ and $36 \mathrm{~h}$ before MCT treatment. The administered volume of vehicle was the same as the volume of regorafenib. Subsequently, some of the rats were sacrificed $48 \mathrm{~h}$ after MCT administration and samples of blood and liver tissue were taken, 
because the histopathological changes at $48 \mathrm{~h}$ after MCT treatment in rats have been found to be most similar to those in human SOS (21). Some animals were sacrificed and samples were collected at two different time points. Partial hepatectomy (30\%, left lateral lobectomy) was performed in SOS rats treated with regorafenib or vehicle (another 15 rats in each group) $48 \mathrm{~h}$ after MCT administration. These animals were monitored for 7 days to examine survival after surgery, while other animals were sacrificed at $24 \mathrm{~h}$ after surgery.

\subsection{Histopathology}

Liver tissue samples were fixed in 4\% paraformaldehyde (PFA), embedded in paraffin wax, and cut into $4 \mu \mathrm{m}$ sections. Histologic assessment of SOS was performed using hematoxylin and eosin (H\&E) staining by a single pathologist (A.M-H.) in a blinded manner. To quantify the degree of SOS, histological changes were scored, as previously reported $(20,21)$. Sinusoidal hemorrhage, endothelial damage in the central vein, and coagulative necrosis of hepatocytes were graded on a 4-point scale: 0 , absent; 1 , mild; 2 , moderate; and 3, severe. The total SOS score was calculated as the sum of the individual scores.

\subsection{Serum biochemistry}

Assessment of biologic data at $48 \mathrm{~h}$ after MCT treatment included serum aspartate transaminase (AST), alanine transaminase (ALT), and albumin (Alb) level measurements.

\subsection{Immunohistochemistry}

Endothelial cells were stained using rat endothelial cell antigen-1 (RECA-1: MCA-970R; AbD Serotec, Oxford, UK), as previously described (21). Tissues were directly embedded in optimal cutting temperature compound (Sakura, Tokyo, Japan). 
Sections were cut at a 6- $\mu \mathrm{m}$ thickness and fixed with $4 \%$ PFA for $10 \mathrm{~min}$ at room temperature. Sections were blocked and then incubated with the primary antibody RECA-1 at a 1:500 dilution for $1 \mathrm{~h}$ at $4^{\circ} \mathrm{C}$. Subsequently, the sections were incubated with labeled polymer using an EnVision + System HRP kit (Dako, Tokyo, Japan), at room temperature for $1 \mathrm{~h}$. The sections were examined after incubation using the Liquid DAB Substrate-Chromogen System (Dako). Ten high-power fields (200×) were randomly selected for calculating the staining area of the endothelial cells. The staining area was quantified using Image J (National Institutes of Health).

For the terminal deoxynucleotidyl transferase-mediated dUTP nick end-loading (TUNEL) assay, we used a commercial kit (In situ Apoptosis Detection Kit; Takara Bio Inc., Shiga, Japan) according to the manufacturer's instructions. TUNEL stained areas were examined in different locations of the tissue section in 10 low-power fields $(100 \times)$ using Image J.

Immunohistochemistry for cleaved caspase-3 was performed using standard protocols with a primary antibody that recognizes cleaved caspase-3 (Asp175) (\#9102, Cell Signaling Technology, Inc. (CST), Danvers, MA, USA). Briefly, the sections were deparaffinized and quenched with hydrogen peroxide, and antigen retrieval was performed using sodium citrate buffer at a sub-boiling temperature for 10 minutes. Subsequently, the sections were incubated with primary antibodies at a 1:400 dilution overnight at $4^{\circ} \mathrm{C}$, followed by incubation with an EnVision + System HRP kit (Dako) for 30 minutes at room temperature. Signals were detected using the Liquid DAB Substrate-Chromogen System (Dako), and the sections were counterstained with hematoxylin. 


\subsection{Electron microscopy}

Rats were first perfused, through the abdominal aorta, with phosphate buffered saline (PBS) and then with a fixative containing 2\% glutaraldehyde and 4\% PFA. The livers were cut into small pieces (approximately $1 \mathrm{~mm}^{3}$ ) for transmission electron microscopy (TEM) and into larger pieces $\left(5 \mathrm{~mm}^{3}\right)$ for scanning electron microscopy (SEM). The samples were fixed in $2 \%$ glutaraldehyde and $4 \%$ PFA at $4{ }^{\circ} \mathrm{C}$. Thin sections were stained with saturated uranyl acetate and lead citrate and were observed using a Hitachi H-7650 electron microscope for TEM. Samples were ion-sputter-coated and observed with a Hitachi S-4700 scanning electron microscope for SEM. The following morphological changes were expected in the liver of rats with SOS: injured SECs with dilated fenestrae resulting in exposure of hepatocytes and penetration of blood cells; detachment of SECs from the sinusoidal wall resulting in enlargement of the space of Disse; and accumulation of blood cells in spaces formally occupied by hepatic sinusoids, reflecting sinusoidal congestion (20).

\subsection{MMP gelatin zymography}

Total protein from the whole liver was purified and $10 \mu \mathrm{g}$ of protein was used in each sample. Gelatin zymography was performed using a Gelatin Zymo electrophoresis kit (Cosmo Bio Co., Tokyo, Japan), according to the manufacturer instructions.

\subsection{Western blot analysis}

Frozen liver tissues were homogenized in lysis buffer containing $10 \mathrm{mmol} / \mathrm{L}$ Tris-HCl (pH 7.4), $150 \mathrm{mmol} / \mathrm{L} \mathrm{NaCl}, 1 \%$ Nonidet P-40, protease inhibitor, and phosphatase inhibitor. Equal amounts of total protein were subjected to $10 \%$ sodium dodecyl sulfate 
polyacrylamide gel electrophoresis, separated by electrophoresis, and transferred onto Immobilon-NC transfer membranes (Millipore Corporation, Billerica, MA, USA). After blocking with 5\% skim milk in TBS-Tween for $1 \mathrm{~h}$, the membranes were incubated with a primary antibody recognizing ERK (\#9102, CST), phosphorylated-specific ERK (\#9101, CST) or GAPDH (sc-25778, Santa Cruz Biotechnology, Inc., Santa Cruz, CA, USA) at a 1:1000 dilution overnight at $4^{\circ} \mathrm{C}$. After treating the membranes with the primary antibodies, a peroxidase-conjugated goat anti-rabbit IgG (sc-2004, Santa Cruz) secondary antibody was used at a 1:2000 dilution for $1 \mathrm{~h}$ at room temperature. Subsequently, bands were detected using ECL reagent (GE Healthcare UK Ltd., Buckinghamshire, UK) and the Ez-Capture II camera (ATTO, Tokyo, Japan). Results were analyzed using Image J.

\subsection{Statistical analysis}

Data were analyzed using JMP 10.0 (SAS Institute, Cary, NC, USA). The Student t-test was used for comparison between groups. Survival after hepatectomy was analyzed using the Kaplan-Meier method, and differences were evaluated using the log-rank test. P values of $<0.05$ were considered statistically significant. Data are expressed as means \pm SD.

\section{Results}

\subsection{Regorafenib attenuates MCT- induced SOS}

In the animals treated with MCT plus vehicle, macroscopic examination at $48 \mathrm{~h}$ after MCT administration showed that the liver surface was irregular, that it had turned dark red in color, and that bloody ascites had accumulated. These findings were less pronounced in the regorafenib group. Microscopically, the liver tissue of the animals in the vehicle group 
displayed the typical features of SOS, with severe sinusoidal hemorrhage, sinusoidal dilatation, coagulative necrosis of hepatocytes, and endothelial damage in the central vein (Fig. 1D and E). Liver sections from the regorafenib group showed a mild degree of SOS, with slight sinusoidal hemorrhage and dilatation compared with the vehicle group (Fig. 1G and $\mathrm{H}$ ). The SOS score in the regorafenib group was significantly lower than that in the vehicle group (Table 1).

With regard to serum biochemistry evaluations at $48 \mathrm{~h}$ after MCT administration, AST and ALT levels were significantly lower in the regorafenib group than in the vehicle group. Furthermore, the Alb level was markedly reduced in the vehicle group compared to the regorafenib group (Table 1). These results suggest that regorafenib attenuated SOS.

\subsection{Regorafenib reduced necrosis in SOS liver}

Hepatocytes undergoing necrosis were detected according to morphologic criteria (vacuolization, loss of structure, karyolysis) with H\&E staining. Moreover, to assess the mode of cell death, the sections were then stained using the TUNEL assay and an antibody against cleaved caspase-3.

In the TUNEL assay, TUNEL-positive cells were overlapped with the necrotic areas, as identified by H\&E staining (Fig. $1 \mathrm{~F}$ and I). However, based on morphological criteria, these cells were not apoptotic. Most TUNEL-positive cells showed nuclear and, especially, cytoplasmic staining, which are both typically observed in cells undergoing oncotic necrosis $(22,23)$. Immunohistochemistry for cleaved caspase-3 revealed that there were only a few positive cells in the necrotic areas, which were detected by H\&E staining and TUNEL staining (Fig. A.1). As caspase-3 activation is one of the most typical 
characteristics observed with apoptosis (23), these results indicated that necrosis was the main constituent of SOS-induced cell death. Thus, we defined the TUNEL-positive area as the area of necrosis, which was significantly decreased in the regorafenib group compared to the vehicle group $(1.40 \% \pm 0.21 \%$ vs. $9.62 \% \pm 0.99 \%$, $\mathrm{p}<0.05$; Fig. $1 \mathrm{~J})$.

\subsection{Regorafenib improves survival after $30 \%$ partial hepatectomy in SOS rats}

To evaluate the effect of regorafenib on remnant liver function after partial hepatectomy in rats with SOS, partial hepatectomy (30\%) was performed in SOS rats treated with regorafenib or vehicle. Kaplan-Meier curves are shown in Fig. 2A. The survival rate at 7 days after surgery was significantly better in the regorafenib group than in the vehicle group ( $26.7 \%$ vs. $6.7 \%$, $p<0.05$ ), indicating that regorafenib improved survival after $30 \%$ hepatectomy in rats with SOS.

To explore the cause of death after hepatectomy, histopathological examinations were performed in both groups at $24 \mathrm{~h}$ after hepatectomy (i.e., $72 \mathrm{~h}$ after MCT administration with hepatectomy). The necrotic areas were further expanded in the vehicle group (Fig. 2B) but not in the regorafenib group (Fig. 2C). When a hepatic resection was not performed, the necrotic areas were absorbed in both groups at the same time point (Fig. 2D and E), which reflected the stage of recovery from liver injury. These results suggested that the hepatic resection promoted the further development of SOS, but that regorafenib may diminish the extent of SOS after hepatectomy, resulting in an improvement of post-operative survival.

\subsection{Regorafenib diminished the loss of SECs due to SOS}

To quantitatively assess the impact of regorafenib on SECs, RECA-1 immunohistochemistry was performed and areas with relative RECA-1 positivity were 
examined (Fig. 3). Compared with untreated rats, areas with relative RECA-1 positivity in the vehicle group significantly decreased with time from $6 \mathrm{~h}$ to $48 \mathrm{~h}$ after MCT administration. In contrast, areas with RECA-1 positivity were significantly larger in the regorafenib group than in the vehicle group both at $6 \mathrm{~h}$ and at $48 \mathrm{~h}$. These results indicated that regorafenib diminished the loss of SECs during the development of SOS.

\subsection{Regorafenib mitigates the SOS-induced morphological damage of SECs as observed on electron microscopy}

To evaluate the morphological changes of SECs in the SOS liver, electron microscopy was performed. SEM showed severe sinusoidal injury in the liver sections of vehicle-treated rats at $48 \mathrm{~h}$ after MCT administration: SECs were completely detached and lost, hepatocytes were directly exposed to sinusoids, and hepatic sinusoids were occupied by red blood cells (Fig. 4A). In the regorafenib group, although SEC injury with enlarged fenestrae was observed, SECs were preserved on the sinusoidal walls (Fig. 4B). On TEM, SECs were completely detached from the sinusoidal walls and blood cells had accumulated between SECs and hepatocytes in vehicle-treated rats (Fig. 4C). In contrast, in regorafenib-treated rats, part of the space of Disse was dilated, but SECs were relatively maintained on parenchymal cells without sinusoidal congestion (Fig. 4D). These results indicated that regorafenib suppressed the morphological injury of SECs during the development of SOS.

\subsection{Regorafenib decreases MMP-9 activity and ERK phosphorylation in SOS livers}

To assess the effect of regorafenib on MMP-9 activity in SOS livers, gelatin zymography was performed. It demonstrated that relative MMP-9 activity at $48 \mathrm{~h}$ after 
MCT treatment in the vehicle group was increased about 6.5 times from that in normal liver, whereas that in the regorafenib group was about 1.7 times higher than normal. Hence, the activity in the regorafenib group at $48 \mathrm{~h}$ after MCT administration was significantly lower than that in the vehicle group (Fig. $5 \mathrm{~A}$ and $\mathrm{B}$ ).

Phosphorylation of ERK was also examined, because it was reported to be necessary for MMP-9 activation (18). According to Western blot analyses of phosphorylated-ERK and total ERK, the relative ratio of p-ERK protein expression to total ERK protein expression in the vehicle group at $6 \mathrm{~h}$ after MCT administration was increased about twofold compared to that of the normal rats $(\mathrm{p}<0.001)$. In contrast, the relative ratio of p-ERK/ERK in the regorafenib group was significantly attenuated compared with the vehicle group $(1.21 \pm 0.32$ vs. $2.09 \pm 0.44, \mathrm{p}<0.01)$ and was equivalent to that of the normal rats (Fig. $5 \mathrm{C}$ and D). These results suggested that regorafenib decreased MMP-9 activation and ERK phosphorylation in SOS liver.

\section{Discussion}

SOS is a form of drug-induced liver injury caused by ingestion of pyrrolidine alkaloids and cytotoxic drugs such as azathioprine, cyclophosphamide, busulfan, and dacarbazine. It was initially considered a complication of high-dose chemotherapy used as conditioning regimens for bone marrow transplantation for hematologic malignancies (24) and is now a well-known contributor to morbidity and mortality after stem cell transplantation (25). For patients with CLM, Rubbia-Brandt et al. first described SOS as a significant hepatic adverse effect of oxaliplatin-based chemotherapy (9). In human studies, although various 
biological markers have been shown to be related to SOS, such as tumor necrosis factor (TNF)-alpha, interleukin (IL)-1b, IL-8, soluble IL-2 receptor, fibrin, fibrinogen, von Willebrand factor, thrombomodulin, and vascular endothelial growth factor (VEGF) $(25,26)$, it was unclear whether these are crucial mediators or consequent features in the process of SOS. Several previous articles have attempted to clarify the precise mechanism of SOS development using a rat model of SOS. DeLeve et al. reported the use of a rat model in which SOS was induced by MCT, a pyrrolizidine alkaloid, with reproducible results (20). The authors have described the mechanism of SOS in their rat model and in vitro as follows (12,20,27). MCT is metabolically activated to MCT pyrrole, which binds to F-actin in SECs and disassembles it. Disassembly of F-actin leads to rounding up of SECs and upregulation of MMP-9. Mechanical stress due to blood flow separates SECs from the underlying parenchymal cells, with subsequent embolization of the sinusoids, and MMP-9 upregulation facilitates degeneration of the extracellular matrix in the space of Disse, expediting SEC detachment from the sinusoidal walls. These microvascular injuries induce typical features of SOS such as sinusoidal dilatation, endothelial damage in the central vein, and coagulative necrosis of hepatocytes.

In the current study, histopathological examination and serum biochemistry revealed that regorafenib attenuated SOS, in concurrence with suppression of ERK phosphorylation and MMP-9 activity. While MMP-9, a collagenase that degrades extracellular matrix and basement membrane components (28), is considered a crucial factor in accelerating the development of SOS, degenerating extracellular matrix in the space of Disse, and promoting the detachment of SECs $(11,12)$, sustained ERK $1 / 2$ phosphorylation is 
necessary for MMP-9 expression (18). In the current study, we first demonstrated that ERK 1/2 was phosphorylated in MCT-induced SOS. Since a previous report indicated that regorafenib inhibits ERK1/2 phosphorylation through the inhibition of the Raf/MEK/ERK pathway (15), we considered that regorafenib attenuated MMP-9 activity through the inhibition of ERK phosphorylation in this study. Previously, we demonstrated that c-Jun N-terminal kinase (JNK) was phosphorylated in the liver after treatment with MCT and that sorafenib reduced the severity of SOS by attenuating the activation of MMP-9 through the inhibition of the phosphorylation of JNK (13). However, another study reported that regorafenib did not inhibit the phosphorylation of JNK (29), suggesting that regorafenib might inhibit the activation of MMP-9, which was independent of JNK. RECA-1 immunostaining in this study showed that SECs were relatively preserved in regorafenib-treated rats compared to vehicle-treated rats at two time points; however, at 48 $\mathrm{h}$ after MCT administration, the rats in the regorafenib group also showed reduced preservation of SECs compared with normal rats. These results suggest that regorafenib indeed significantly attenuated the loss of SECs from the sinusoidal walls, but did not completely prevent this loss. During the development of SOS, the embolization of detached SECs induces congestion of the sinusoid and microcirculatory disturbance, resulting in the typical histopathological features of SOS. Because regorafenib reduces the detachment of SECs in the early phase, it might lead to an improvement in the severity of SOS in the advanced phase. Electron microscopic evaluation in this study also showed that enlargement of the space of Disse and separation of SECs from parenchymal cells were suppressed in the regorafenib group, whereas SECs were detached from the underlying 
parenchymal cells and drained away in the vehicle group. These effects for the preservation of SECs in the regorafenib group might have been induced by suppression of the ERK/MMP-9-related pathway, which promotes SOS and is a crucial but not the sole mechanism in the loss of SECs. Moreover, Topp et al. reported that MMP-9 itself was involved in the morphological injury of SECs, in a manner related to actin disassembly, although actin disassembly has been shown to be necessary for the secretion of MMP-9 (30). Electron microscopic images in our study also showed that the morphology of SECs was comparatively preserved in regorafenib-treated rats, with suppression of MMP-9 activity. MMP-9 might independently promote morphologic SEC damage, in addition to inducing extracellular matrix degeneration.

Associated with recent developments in chemotherapy for colorectal cancer, newly developed drugs including oxaliplatin produce high response rates in patients with CLM, ranging from $43 \%$ to $60 \%(31,32,33)$, compared with the rates of traditional drugs, which range from $18 \%$ to $30 \%(34,35)$. A recent study reported that $22.5 \%$ of patients with initially unresectable CLM underwent curative resection following neoadjuvant chemotherapy and that their overall survival was comparable to that of patients with resectable CLM (36). The incidence of hepatic resection following neoadjuvant chemotherapy in patients with CLM may therefore increase in the future. In patients with CLM, SOS is detected in 51-64\% of patients after oxaliplatin-based chemotherapy $(9,37,38)$ and is a serious concern because of its adverse effects. Vreuls et al. reported that a higher grade of SOS was associated with a lower tumor response to neoadjuvant oxaliplatin treatment (38). Nakano et al. reported that SOS increased morbidity after major 
hepatectomy in patients with CLM (10). Tamandl et al. showed that SOS led to early recurrence and decreased survival in the long term (39). These reports highlight the importance of a strategy for the prevention of SOS. To date, several studies have demonstrated that bevacizumab, a humanized anti-VEGF monoclonal antibody, has a protective effect against oxaliplatin-induced SOS $(40,41,42)$; however, the mechanism of prevention is still unclear and the validity of this effect has been questioned (43). In a rat model of SOS, MMP inhibitors, phosphodiesterase (PDE) III inhibitors, and sorafenib have been reported to prevent SOS (12,13,21); however, MMP inhibitors and PDE III inhibitors are not widely used to treat SOS in humans. Sorafenib is a multikinase inhibitor approved for the treatment of hepatocellular carcinoma and renal cell carcinoma around the world, although it is not used in combination with oxaliplatin for colorectal cancer at present, and the probability of its future application is low because of the unsatisfactory results obtained in clinical studies (14).

Regorafenib is the first small-molecule kinase inhibitor to show efficacy against metastatic colorectal cancer (16). Currently, it is approved for metastatic colorectal cancer as monotherapy; furthermore, it potentially has additional therapeutic value as combined therapy with oxaliplatin as first- or second-line therapy, based on the results of clinical trials (17). Our results suggested that hepatic resection may cause shear stress on the SOS liver, which may accelerate the loss of SECs, resulting in the further development of SOS and death after hepatic resection. Because regorafenib suppresses SOS at the time of hepatectomy, regorafenib might diminish the deterioration caused by SOS after hepatectomy and improve post-operative survival. Based on the findings of our experiments, 
if combined treatment with regorafenib and oxaliplatin is approved as being effective for CLM, this combination is potentially desirable from two viewpoints. First, the addition of regorafenib to oxaliplatin treatment would bring the advantage of an anticancer effect, and the intense pharmacological effect might allow patients with initially unresectable CML to undergo hepatectomy with the intent to cure. Second, the addition of regorafenib would suppress oxaliplatin-induced SOS, help reduce morbidity, and enable additional patients with unresectable CML due to insufficient remnant liver function to become candidates for curative hepatic resection. However, it is premature to directly apply our findings from an animal study to humans. Hence, further studies and the results from human clinical trials are needed before we arrive at a definitive conclusion.

There are several limitations in our study. First, the precise mechanism of SOS and the manner in which regorafenib mitigates SOS have not yet been precisely clarified. We may have only demonstrated one of the possible pathways because regorafenib is a multi-kinase inhibitor that may affect various kinase pathways that are also involved in SOS development. Second, the rat SOS model that we used in this study has been reported to mimic the histopathological features of human SOS $(20,21)$. Because MCT-induced liver injury was acute and severe, this animal model may be somewhat different from oxaliplatin-induced SOS in humans. Therefore, further investigations and clinical evaluations are necessary.

In conclusion, the results of the present study show that regorafenib diminishes MCT-induced SOS in rats, concomitant with the inhibition of ERK phosphorylation and MMP-9 activation. Pretreatment with regorafenib also resulted in a significant improvement 
in survival following hepatectomy in rats with SOS. These results suggest that pretreatment with regorafenib may constitute a therapeutic strategy to prevent SOS in patients with CLM in addition to facilitating an antitumor effect. Data from ongoing clinical trials may help to confirm our findings.

\section{Acknowledgments}

We are grateful to Bayer HealthCare Pharmaceuticals Inc. (Whippany, NJ) for their kind gift of regorafenib. We also acknowledge the support of Ms. Keiko Okamoto-Furuta and Mr. Haruyasu Kohda from the Division of Electron Microscopic Study, Center for Anatomical Studies, Graduate School of Medicine, Kyoto University. We received no financial support from any society for this study.

\section{References}

1) Van Cutsem E, Rivera F, Berry S, Kretzschmar A, Michael M, DiBartolomeo M, et al. Safety and efficacy of first-line bevacizumab with FOLFOX, XELOX, FOLFIR and fluoropyrimidines in metastatic colorectal cancer: the BEAT study. Ann Oncol 2009;20:1842-7.

2) Eisenberg B, Decosse JJ, Harford F, Michalek J. Carcinoma of the colon and rectum: the natural history reviewed in 1704 patients. Cancer 1982;49:1131-4.

3) Steele GJ, Ravikumar TS. Resection of hepatic metastases from colorectal metastases: biologic perspectives. Ann Surg 1989;210:127-38.

4) Jaeck D, Bachellier P, Guiguet M, Boudjema K, Vaillant JC, Balladur P, et al. 
Long-term survival following resection of colorectal hepatic metastases. $\mathrm{Br} \mathrm{J}$ Surg 1997; 84: 977-980.

5) Scheele J, Stang R, Altendorf-Hofmann A, Paul M. Resection of colorectal liver metastases. World J Surg 1995;19:59-71.

6) Stangl R, Alterndorf-Hofmann A, Charnley RM, Scheele J. Factors influencing the natural history of colorectal liver metastases. Lancet 1994;343:1405-10.

7) Adam R. Developing Strategies for Liver Metastases From Colorectal Cancer. Semin Oncol 2007;34:S7-11

8) Vauthey JN, Pawlik TM, Ribero D, Wu TT, Zorzi D, Hoff PM, et al. Chemotherapy regimen predicts steatohepatitis and an increase in 90-Day mortality after surgery for hepatic colorectal metastases. J Clin Oncol 2006;24:2065-72.

9) Rubbia-Brandt L, Audard V, Sartoretti P, Roth AD, Brezault C, Le Charpentier M, et al. Severe hepatic sinusoidal obstruction associated with oxaliplatinbased chemotherapy in patients with metastatic colorectal cancer. Ann Oncol 2004;15:460-6.

10) Nakano H, Oussoultzoglou E, Rosso E, Casnedi S, Chenard-Neu MP, Dufour P, et al. Sinusoidal Injury Increases Morbidity After Major Hepatectomy in Patients With Colorectal Liver Metastases Receiving Preoperative Chemotherapy. Ann Surg 2008;247:118-24.

11) Hanumegowda UM, Copple BL, Shibuya M, Malle E, Ganey PE, Roth RA. Basement membrane and matrix metalloproteinases in monocrotaline-induced liver injury. Toxicol Sci 2003;76:237-46.

12) Deleve LD, Wang X, Tsai J, Kanel G, Strasberg S, Tokes ZA. Sinusoidal obstruction 
syndrome (veno-occlusive disease) in the rat is prevented by matrix metalloproteinase inhibition. Gastroenterology 2003;125:882-90.

13) Nakamura K, Hatano E, Narita M, Miyagawa-Hayashino A, Koyama Y, Nagata H, et al. Sorafenib attenuates monocrotaline-induced sinusoidal obstruction syndrome in rats through suppression of JNK and MMP-9. J Hepatol 2012;57:1037-43.

14) Tabernero J, Garcia-Carbonero R, Cassidy J, Sobrero A, Van Cutsem E, Köhne CH, et al. Sorafenib in Combination with Oxaliplatin, Leucovorin, and Fluorouracil (Modified FOLFOX6) as First-line Treatment of Metastatic Colorectal Cancer: The RESPECT Trial. Clin Cancer Res 2013;19:2541-50.

15) Wilhelm SM, Dumas J, Adnane L, Lynch M, Carter CA, Schütz G, et al. Regorafenib (BAY 73-4506): a new oral multikinase inhibitor of angiogenic, stromal and oncogenic receptor tyrosine kinases with potent preclinical antitumor activity. Int J Cancer 2011;129:245-55.

16) Grothey A, Van Cutsem E, Sobrero A, Siena S, Falcone A, Ychou M, et al. Regorafenib monotherapy for previously treated metastatic colorectal cancer (CORRECT): an international, multicentre, randomised, placebo-controlled, phase 3 trial. Lancet 2013;381:303-12.

17) Schultheis B, Folprecht G, Kuhlmann J, Ehrenberg R, Hacker UT, Köhne CH, et al. Regorafenib in combination with FOLFOX or FOLFIRI as first- or second-line treatment of colorectal cancer: results of a multicenter, phase Ib study. Ann Oncol 2013;24:1560-7.

18) Genersch E, Hayess K, Neuenfeld Y, Haller H. Sustained ERK phosphorylation is 
necessary but not sufficient for MMP-9 regulation in endothelial cells: involvement of Ras-dependent and -independent pathways. J Cell Sci 2000;113:4319-30.

19) Prié S, Stewart DJ, Dupuis J. Endothelin A receptor blockade improves nitric oxide-mediated vasodilation in monocrotaline-induced pulmonary hypertension. Circulation 1998;97:2169-74.

20) DeLeve LD, McCuskey RS, Wang X, Hu L, McCuskey MK, Epstein RB, et al. Characterization of a reproducible rat model of hepatic veno-occlusive disease. Hepatology 1999;29:1779-91.

21) Narita M, Hatano E, Ikai I, Miyagawa-Hayashino A, Yanagida A, Nagata H, et al. A phosphodiesterase III inhibitor protects rat liver from sinusoidal obstruction syndrome through heme oxygenase-1 induction. Ann Surg 2009;249:806-13.

22) Hong JY, Lebofsky M, Farhood A, Jaeschke H. Oxidant stress-induced liver injury in vivo: role of apoptosis, oncotic necrosis, and c-Jun NH2-terminal kinase activation. Am J Physiol Gastrointest Liver Physiol. 2009;296:G572-81.

23) Jaeschke H, Lemasters JJ. Apoptosis versus oncotic necrosis in hepatic ischemia/reperfusion injury. Gastroenterology. 2003;125:1246-57.

24) Coppell JA, Richardson PG, Soiffer R, Martin PL, Kernan NA, Chen A, et al. Hepatic Veno-Occlusive Disease following Stem Cell Transplantation: Incidence, Clinical Course, and Outcome. Biol Blood Marrow Transplant 2010;16:157-68.

25) Coppell JA, Brown SA, Perry DJ. Veno-occlusive disease: cytokines, genetics, and haemostasis. Blood Rev 2003;17: 63-70.

26) Iguchi A , Kobayashi R, Yoshida M, Kobayashi K, Matsuo K, Kitajima I, et al. Vascular 
endothelial growth factor (VEGF) is one of the cytokines causative and predictive of hepatic veno-occlusive disease (VOD) in stem cell transplantation. Bone Marrow Transplan 2001;27:1173-80.

27) DeLeve LD, Ito Y, Bethea NW, McCuskey MK, Wang X, McCuskey RS. Embolization by sinusoidal lining cells obstructs the microcirculation in rat sinusoidal obstruction syndrome. Am J Physiol Gastrointest Liver Physiol 2003;284:G1045-52.

28) Hulboy DL, Rudolph LA, Matrisian LM. Matrix metalloproteinases as mediators of reproductive function. Mol Hum Reprod 1997;3:27-45.

29) Carr BI, Cavallini A, Lippolis C, D'Alessandro R, Messa C, Refolo MG, et al. Fluoro-Sorafenib (Regorafenib) effects on hepatoma cells: growth inhibition, quiescence, and recovery. J Cell Physiol. 2013;228:292-7.

30) Topp SA, Upadhya GA, Strasberg SM. Cold preservation of isolated sinusoidal endothelial cells in MMP 9 knockout mice: effect on morphology and platelet adhesion. Liver Transpl 2004;10:1041-8.

31) Giacchetti S, Itzhaki M, Gruia G, Adam R, Zidani R, Kunstlinger F, et al. Long-term survival of patients with unresectable colorectal cancer liver metastases following infusional chemotherapy with 5-fluorouracil, leucovorin, oxaliplatin and surgery. Ann Oncol 1999;10:663-9.

32) Alberts SR, Horvath WL, Sternfeld WC, Goldberg RM, Mahoney MR, Dakhil SR, et al. Oxaliplatin, fluorouracil, and leucovorin for patients with unresectable liver-only metastases from colorectal cancer: a North Central Cancer Treatment Group phase II study. J Clin Oncol 2005; 23: 9243-49. 
33) Nuzzo G, Giuliante F, Ardito F, Vellone M, Pozzo C, Cassano A, et al. Liver resection for primarily unresectable colorectal metastases downsized by chemotherapy. J Gastrointest Surg 2007;11:318-24.

34) Petrelli N, Douglass HO Jr, Herrera L, Russell D, Stablein DM, Bruckner HW, et al. The modulation of fluorouracil with leucovorin in metastatic colorectal carcinoma: A prospective randomized phase III trial. J Clin Oncol 1989; 7: 1419-26.

35) Maiello E, Gebbia V, Giuliani F, Paoletti G, Gebbia N, Cigolari S, et al. 5-Fluorouracil and folinic acid with or without CPT-11 in advanced colorectal cancer patients: a multicenter randomised phase II study of the Southern Italy Oncology Group. Ann Oncol 2000;11:1045-51.

36) Lam VW, Spiro C, Laurence JM, Johnston E, Hollands MJ, Pleass HC, et al. A Systematic Review of Clinical Response and Survival Outcomes of Downsizing Systemic Chemotherapy and Rescue Liver Surgery in Patients with Initially Unresectable Colorectal Liver Metastases. Ann Surg Oncol 2012;19:1292-301.

37) van den Broek MA, Vreuls CP, Winstanley A, Jansen RL, van Bijnen AA, Dello SA, et al. Hyaluronic Acid as a Marker of Hepatic Sinusoidal Obstruction Syndrome Secondary to Oxaliplatin-Based Chemotherapy in Patients with Colorectal Liver Metastases. Ann Surg Oncol 2013;20:1462-9

38) Vreuls CP, Van Den Broek MA, Winstanley A, Koek GH, Wisse E, Dejong CH, et al. Hepatic sinusoidal obstruction syndrome (SOS) reduces the effect of oxaliplatin in colorectal liver metastases. Histopathology 2012;61:314-8.

39) Tamandl D, Klinger M, Eipeldauer S, Herberger B, Kaczirek K, Gruenberger B, et al. 
Sinusoidal obstruction syndrome impairs long-term outcome of colorectal liver metastases treated with resection after neoadjuvant chemotherapy. Ann Surg Oncol 2011;18:421-30.

40) Ribero D, Wang H, Donadon M, Zorzi D, Thomas MB, Eng C, et al. Bevacizumab improves pathologic response and protects against hepatic injury in patients treated with oxaliplatin-based chemotherapy for colorectal liver metastases. Cancer 2007;110:2761-7.

41) Klinger M, Eipeldauer S, Hacker S, Herberger B, Tamandl D, Dorfmeister M, et al. Bevacizumab protects against sinusoidal obstruction syndrome and does not increase response rate in neoadjuvant XELOX/FOLFOX therapy of colorectal cancer liver metastases. Eur J Surg Oncol 2009;35:515-20.

42) van der Pool AE, Lalmahomed ZS, de Wilt JH, Eggermont AM, Ijzermans JM, Verhoef C. Effect of Bevacizumab Added Preoperatively to Oxaliplatin on Liver Injury and Complications After Resection of Colorectal Liver Metastases. J Gastrointest Surg 2009;13:890-5.

43) Zalinski S, Bigourdan JM, Vauthey JN. Does bevacizumab have a protective effect on hepatotoxicity induced by chemotherapy? J Chir 2010;147:S18-24. 


\section{Figure Legends}

Fig. 1 Effect of regorafenib on liver pathology in monocrotaline-induced sinusoidal obstruction syndrome in rats. Hematoxylin and eosin $(\mathrm{H} \& \mathrm{E})$ staining of a normal rat liver (A: 40×, B: 200×). H\&E staining in a vehicle-treated rat showed sinusoidal hemorrhage and dilatation (arrow head), coagulative necrosis of hepatocytes (white arrow), and endothelial damage of the central vein (black arrow) (D: 40×, E: 200×). H\&E staining in a regorafenib-treated rat (G: 40×, H: 200×). No TUNEL-positive cells were identified in the normal rat samples (C). The TUNEL-positive cells coincided with the necrotic areas identified by $H \& E$ staining in a vehicle-treated rat $(F)$ and in a regorafenib-treated rat (I). (J) Areas of relative necrosis at different locations of the tissue section in 10 low-power fields ( $n$ = 9 in each group). MCT, monocrotaline; VHC, vehicle; REGO, regorafenib; *p < 0.05 .

Fig. 2 Effect of regorafenib on survival after partial hepatectomy. (A) Kaplan-Meier curves for 7 days after partial hepatectomy (30\%) in the regorafenib group (gray line) and vehicle group (black line) ( $\mathrm{n}=15$ in each group). The survival rate at 7 days after surgery was significantly higher in the regorafenib group than in the vehicle group (26.7\% vs. 6.7\%, $\mathrm{p}<0.05)$. Hematoxylin and eosin $(\mathrm{H} \& \mathrm{E})$ staining $(100 \times)$ in a vehicle-treated rat at $24 \mathrm{~h}$ after hepatectomy (i.e., at $72 \mathrm{~h}$ after MCT administration) (B) showed further expansion of the necrotic area, unlike in a regorafenib-treated rat (C). H\&E staining $(100 \times)$ in a vehicle-treated rat (D) and in a regorafenib-treated rat (E) at $72 \mathrm{~h}$ after MCT administration without hepatectomy showed the absorption of the necrotic areas.

Fig. 3 Effect of regorafenib on the loss of sinusoidal endothelial cells in sinusoidal 
obstruction syndrome rats. (A) Areas of relative RECA-1 positivity at different locations of the tissue section in 10 high-power fields $(200 \times)$ at $6 \mathrm{~h}$ and $48 \mathrm{~h}$ after MCT administration in the regorafenib group (gray line) and vehicle group (black line) (n = 5 in each). ${ }^{*} \mathrm{p}<0.05$ between the two groups; \#p $<0.05$ compared with normal rats. (B) Representative images of immunohistochemistry for RECA-1 in a normal rat and in a rat treated with regorafenib or vehicle at $6 \mathrm{~h}$ and $48 \mathrm{~h}$ after MCT administration. RECA-1, rat endothelial cell antigen 1; MCT, monocrotaline; VHC, vehicle; REGO, regorafenib.

Fig. 4 Electron microscopy images. (A) SEM in the vehicle group (2000×). (B) SEM in the regorafenib group $(3000 \times)$. (C) TEM in the vehicle group $(2000 \times)$. (D) TEM in the regorafenib group (2000×). All samples were collected at $48 \mathrm{~h}$ after MCT treatment. The scale bars correspond to $2 \mu \mathrm{m}$. The arrow indicates an enlarged fenestration in SECs. S, sinusoidal endothelial cell; D, space of Disse; MCT, monocrotaline; VHC, vehicle; REGO, regorafenib; SEM, scanning electron microscopy; TEM, transmission electron microscopy.

Fig. 5 Activity of MMP-9 and expression of ERK and phosphorylated ERK. (A) The relative MMP-9 activity in the liver tissue from gelatin zymography quantified by densitometry in the regorafenib group and vehicle group at $48 \mathrm{~h}$ after MCT treatment ( $\mathrm{n}=5$ in each group). (B) Representative image of gelatin zymography in a regorafenib-treated rat and in a vehicle-treated rat. (C) The relative ratio of p-ERK/ERK in the liver tissue based on western blot analysis and quantified by densitometry in the regorafenib group and vehicle group at $6 \mathrm{~h}$ after MCT treatment ( $\mathrm{n}=5$ in each group). (D) Representative images of western blot analysis of ERK, p-ERK and GAPDH in a regorafenib-treated rat and in a vehicle-treated rat. MMP-9, matrix metalloproteinase-9; ERK, extracellular 
signal-regulated kinase; p-ERK, phosphorylated extracellular signal-regulated kinase; MCT, monocrotaline; VHC, vehicle; REGO, regorafenib; N.S., not significant; ${ }^{*} \mathrm{p}<0.05$, **p < 0.001 .

Fig. A.1 Immunostaining of cleaved caspase-3. No cleaved caspase 3-positive cells were identified in liver from a normal rat (A). Only a few cells were identified as positive for cleaved caspase-3 (arrow) in the necrotic areas in a vehicle-treated rat (B) and in a regorafenib-treated rat (C). Original magnification was 200×. 


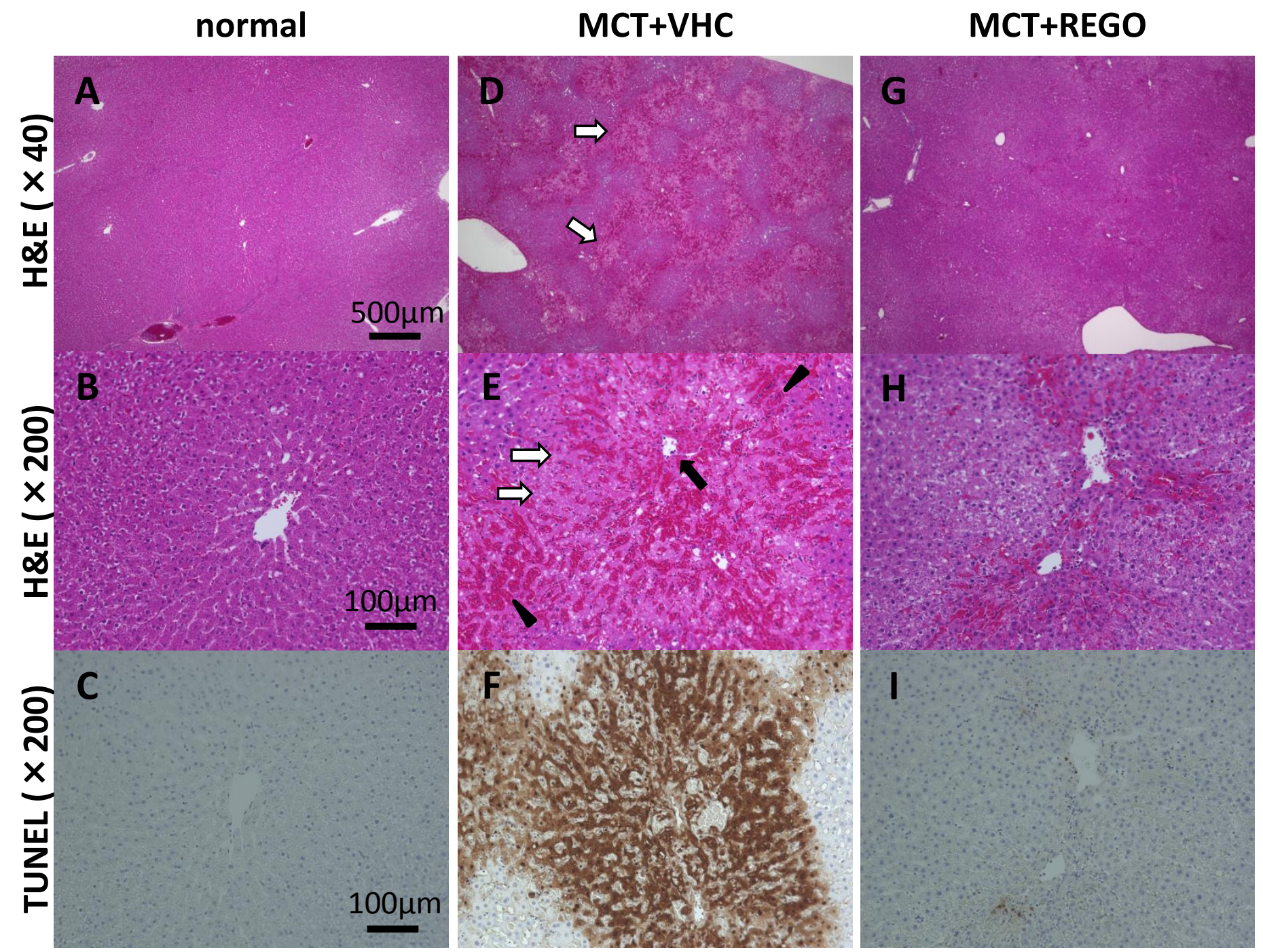

J

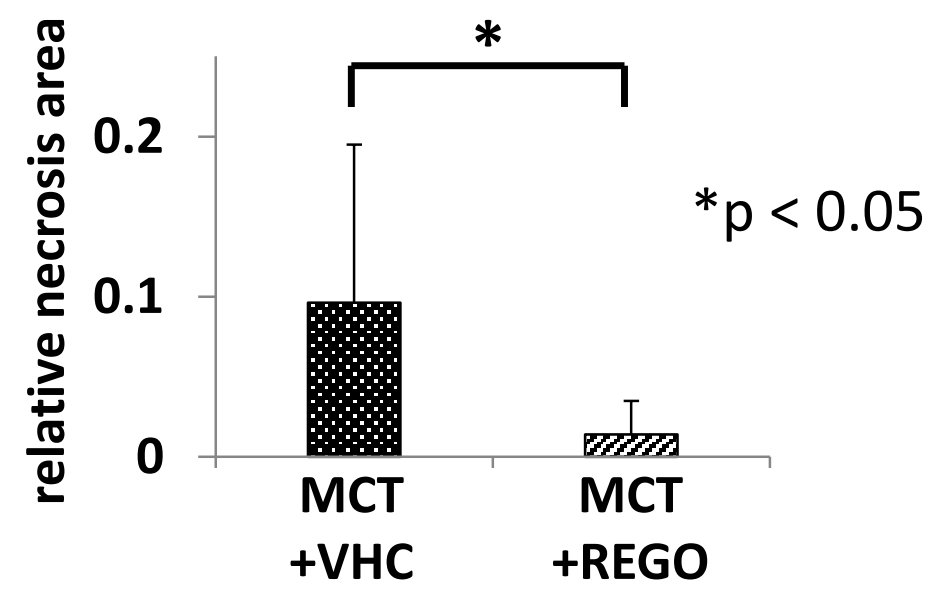


A

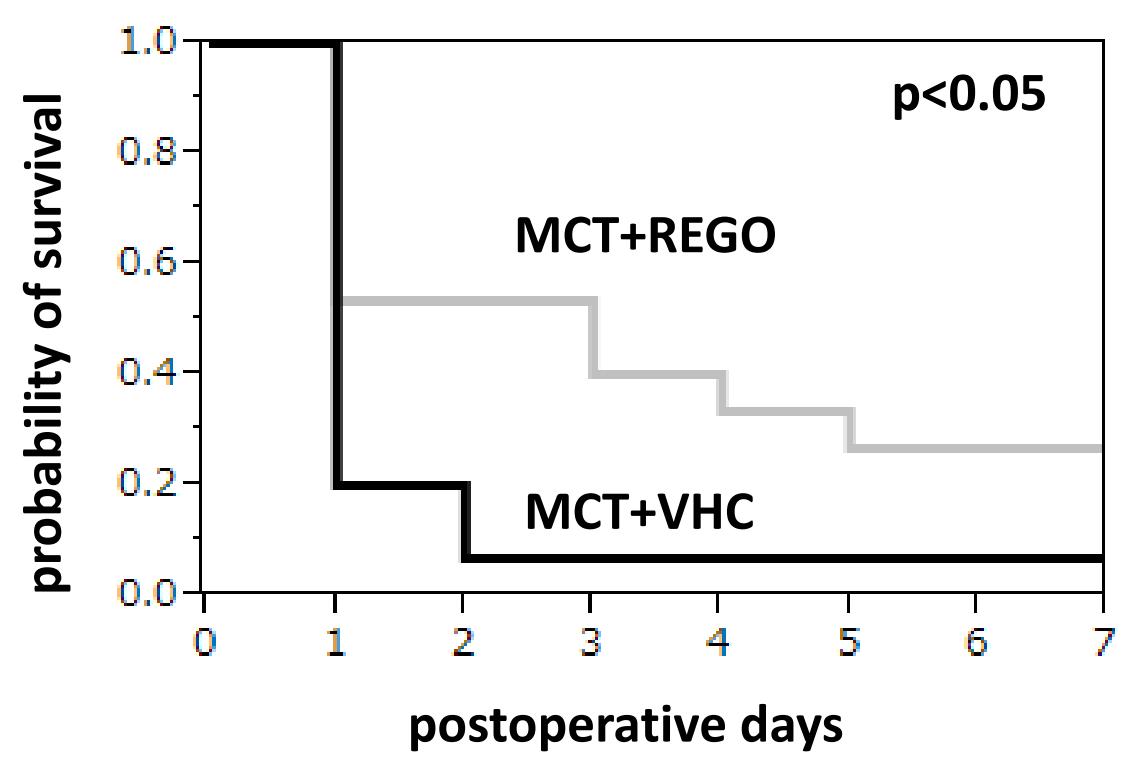

72h (+hepatectomy)
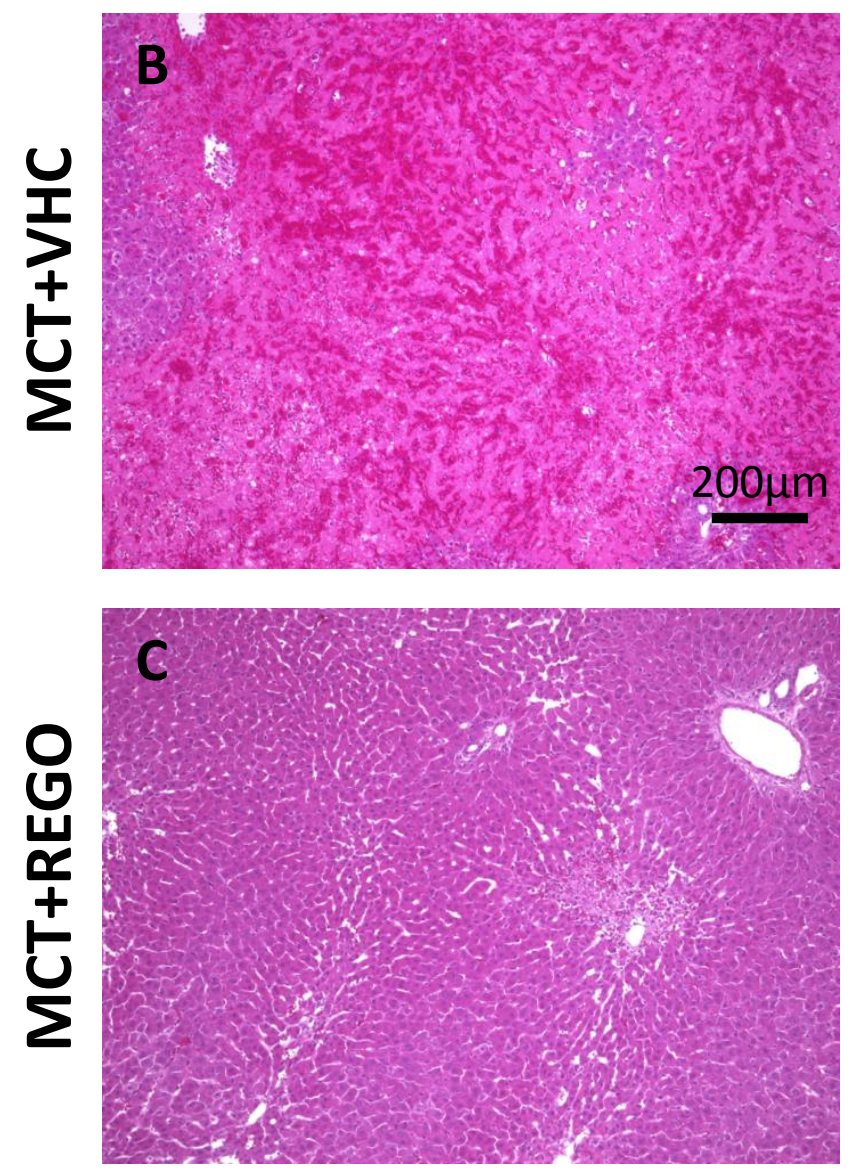

72h (-hepatectomy)
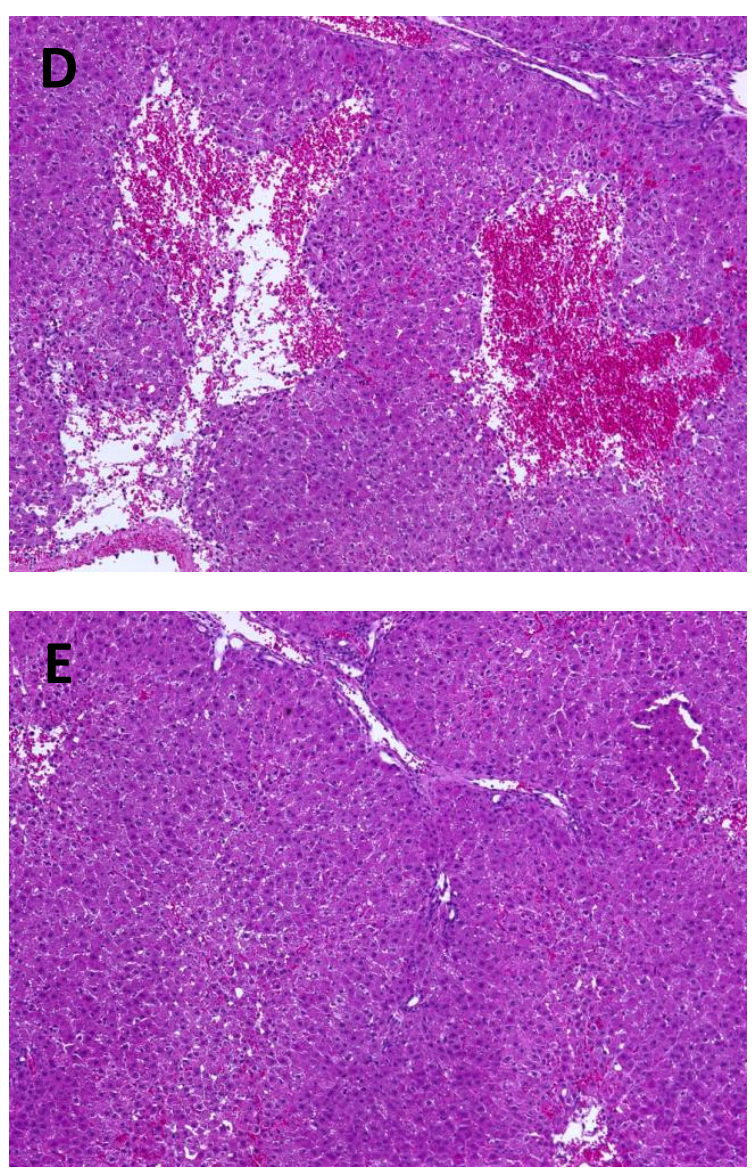
A

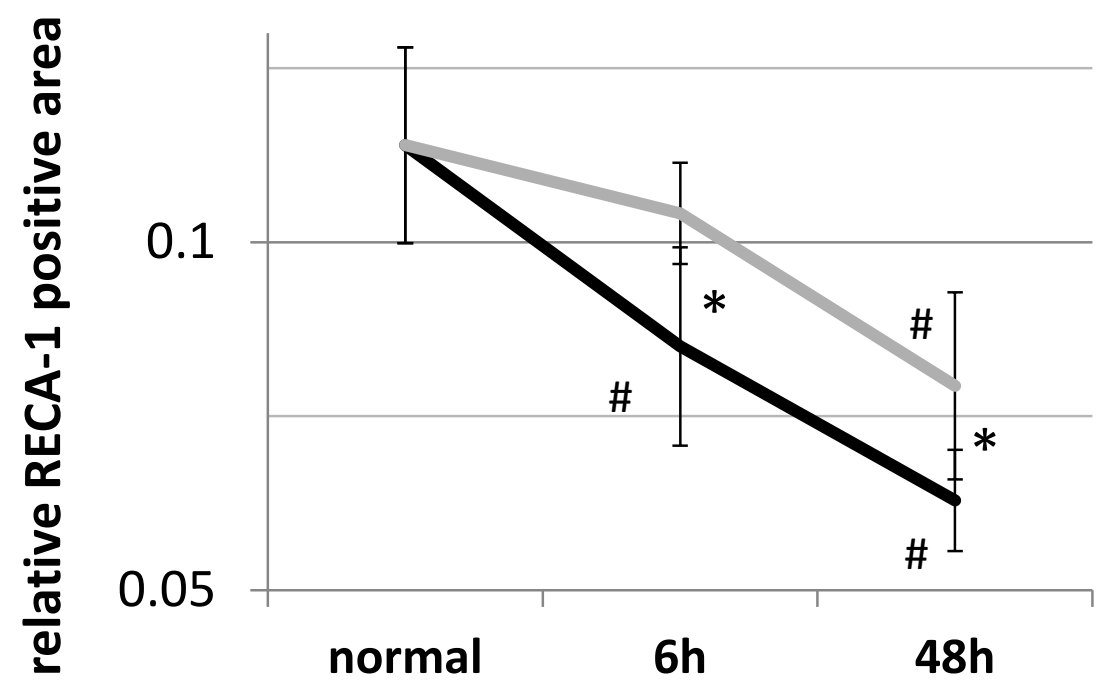

$\longrightarrow \mathrm{MCT}+\mathrm{VHC}$

MCT+REGO

${ }^{*} p<0.05$ compared between each group $\# p<0.05$ compared with normal

B
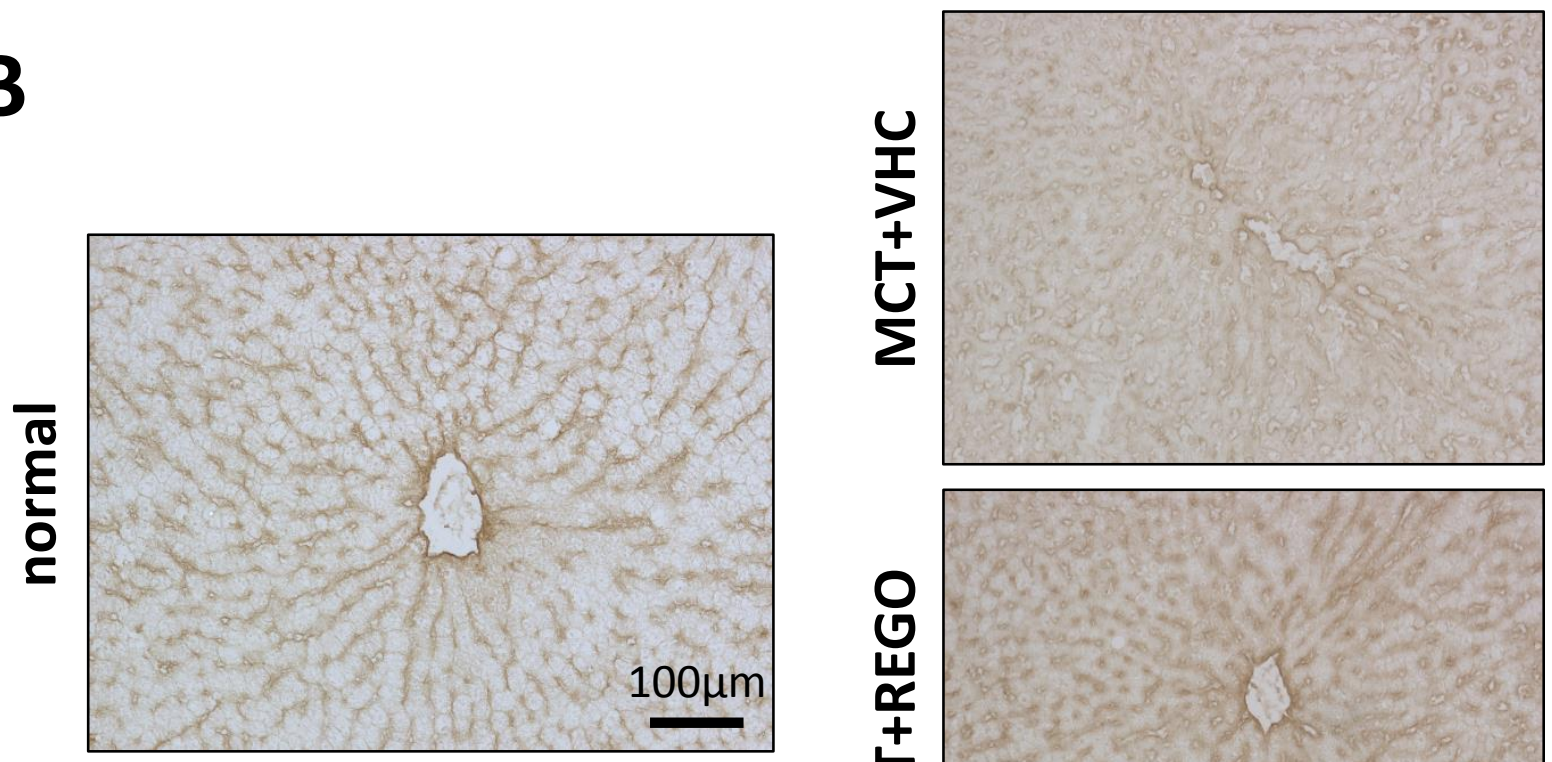

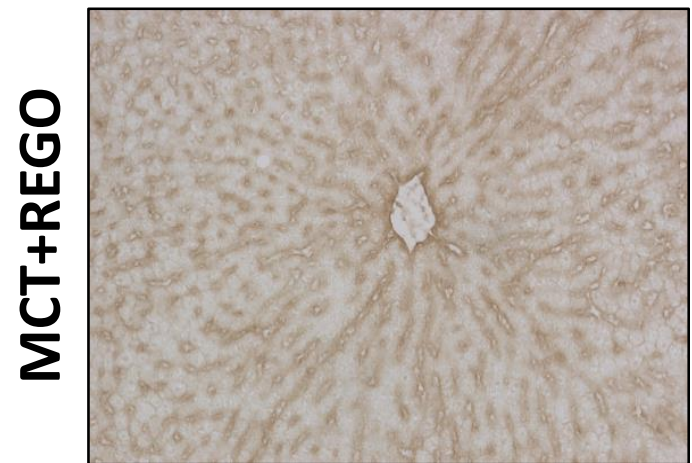

$6 h$
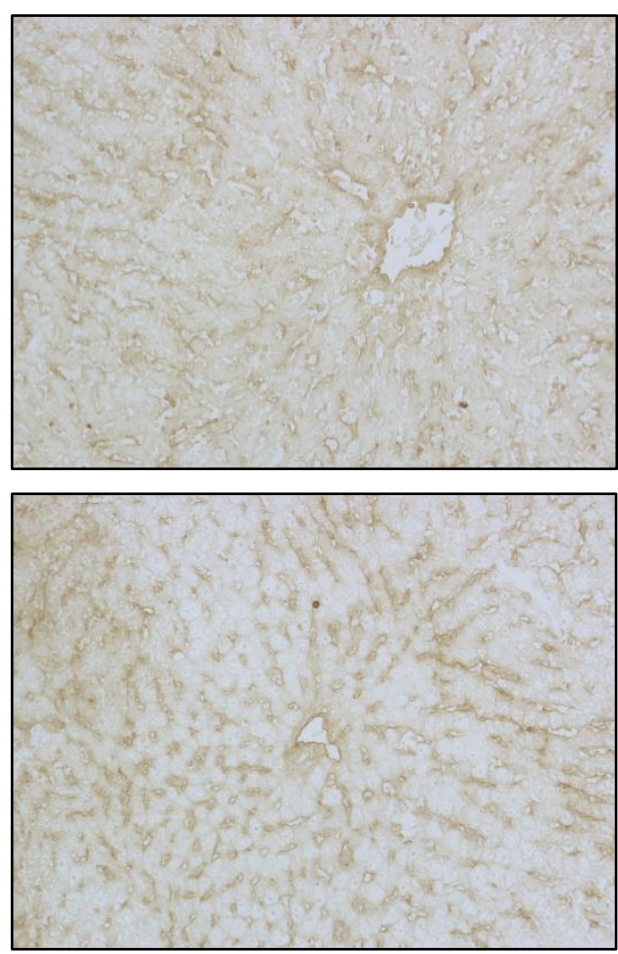

$48 h$ 
A

\section{$\mathrm{MCT}+\mathrm{VHC}$}

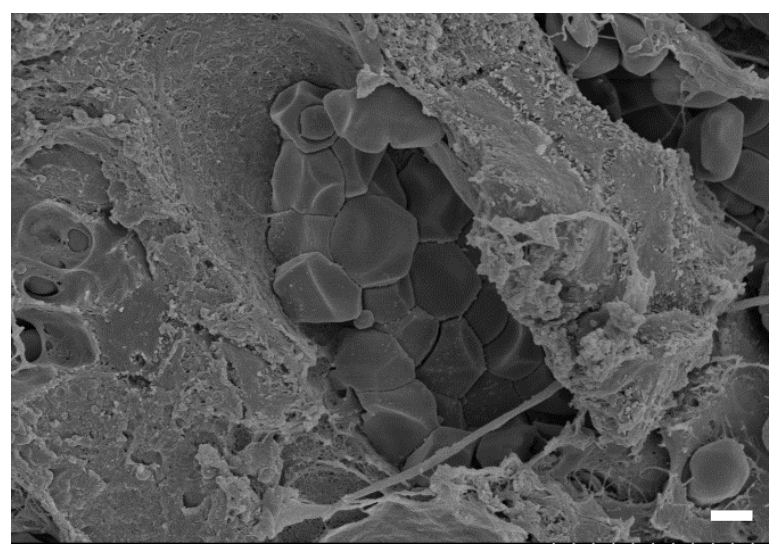

C

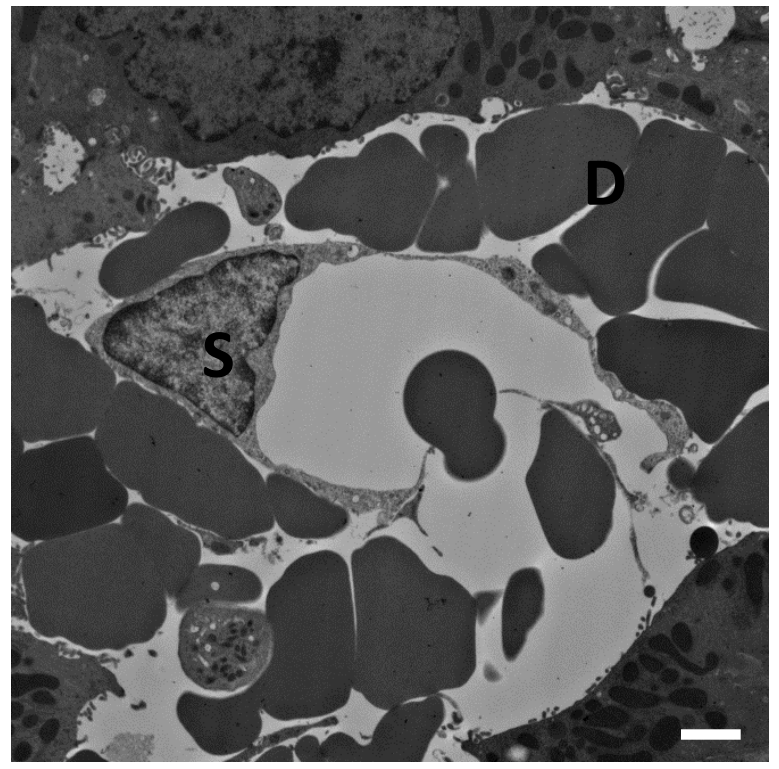

B

\section{MCT+REGO}

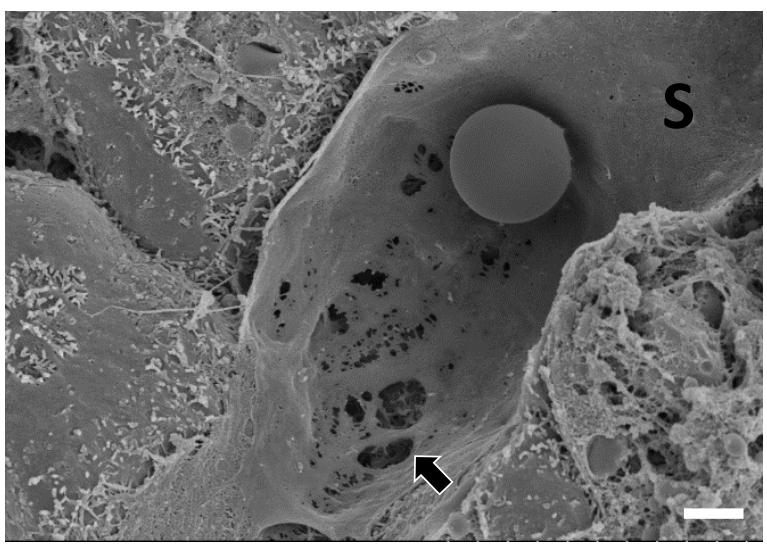

D

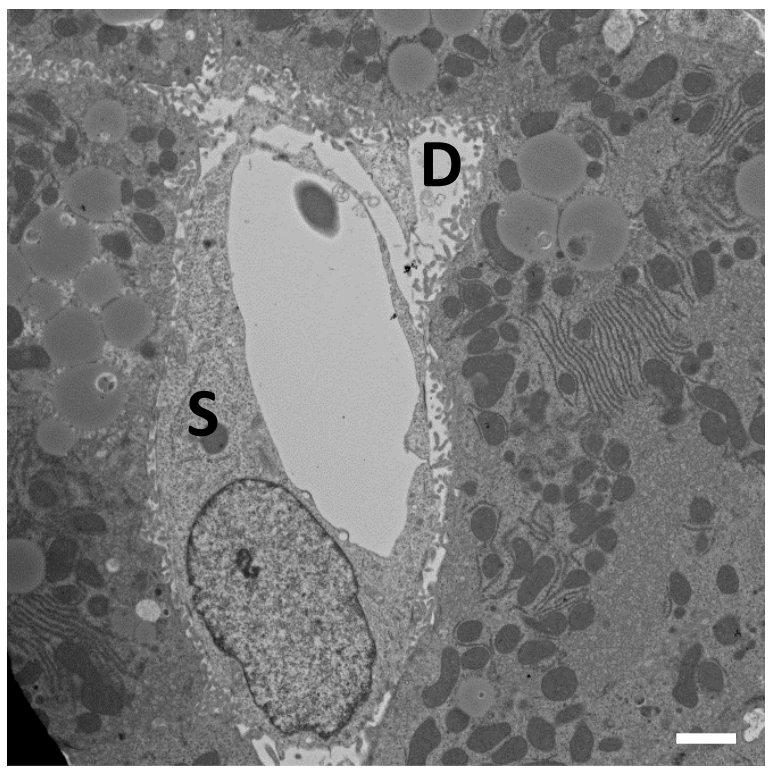


normal

A

$100 \mu \mathrm{m}$
MCT+VHC

B

$\searrow$

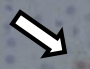

MCT+REGO

C

S 
Table 1 SOS score for HE staining and serum biochemistry at $48 \mathrm{~h}$ after MCT administration

\begin{tabular}{cccc}
\hline & MCT+vehicle & MCT+regorafenib & p \\
\hline SOS score & $8.20 \pm 1.36$ & $7.05 \pm 1.96$ & $<0.05$ \\
AST [IU/L] & $5484 \pm 2685$ & $3100 \pm 1400$ & $<0.01$ \\
ALT [IU/L] & $2389 \pm 1159$ & $1736 \pm 798$ & $<0.05$ \\
Alb [g/dl] & $3.10 \pm 0.21$ & $3.29 \pm 0.20$ & $<0.01$ \\
\hline
\end{tabular}

Data are shown as means \pm SD. $n=20$ in each group

SOS, sinusoidal obstruction syndrome; HE, hematoxylin and eosin; MCT, monocrotaline; AST, aspartate aminotransferase; ALT, alanine aminotransferase; Alb, albmine. 